\title{
Metodologias ativas de ensino-aprendizagem: perfil e capacitação pedagógica do docente do curso de medicina
}

\author{
Active methodologies of teaching-learning: profile and \\ pedagogical training of teachers of medical school
}

\author{
Lizomar de Jesus Maués Pereira Moia1,2, Raphael Primo Martins de Sousa, \\ Renato Mauro Vieira Souza ${ }^{3}$, Aline Batista da Fonseca ${ }^{3}$ \\ 'Hospital Fundação Santa Casa de Misericórdia do Pará, Belém (PA), Brasil \\ 2Universidade do Estado do Pará, Belém (PA), Brasil. \\ ${ }^{3}$ Universidade do Estado do Pará, Campus XII, Santarém (PA), Brasil.
}

Recebido: Maio 31, 2016 Aceito: Nov. 20, 2017

\section{COMO CITAR ESTE ARTIGO} Moia LJMP, Sousa RPM, Souza RMV, Fonseca AB. Metodologias ativas de ensino-aprendizagem: perfil e capacitaça pedagógica do docente do curso de medicina. Interdisciplinary Journal of Health Education. 2017 Jan-Jul;2(1):1-9. https://doi.org/10.4322/ijhe.2016.012

\section{CORRESPONDÊNCIA}

Lizomar de Jesus Maués Pereira Móia Seção de Hepatologia, Instituto Evandro Chagas

Av. Almirante Barroso, 492, CEP 66090-000, Belém (PA), Brasil Tel. (91) 211-4461

lizmoia@yahoo.com.br

\section{FONTE DE FINANCIAMENTO}

Conselho Nacional de Desenvolvimento Científico e Tecnológico- CNPq.

\section{CONFLITO DE INTERESSE}

Os autores declararam não

haver conflitos de interesse.

O estudo foi realizado em Santarém (PA), Brasil.

Todos os autores leram e aprovam a versão final submetida ao Interdisciplinary Journal of Health Education (IJHE).

\section{RESUMO}

O corpo docente é a base fundamental sobre a qual devem ser instituídas as mudanças necessárias para a formação da área da saúde. Diversos estudos têm constatado a usência de preparo pedagógico do professor de medicina, em especial, às metodologias ativas de ensino e aprendizagem. Objetivo: Este artigo traçou o perfil profissional dos professores do curso de medicina da Universidade do Estado do Pará (UEPA) em Santarém, e conhecer o seu nível de capacitação acerca das metodologias de ensino médico, com ênfase no método de Aprendizagem Baseado em Problemas (ABP). Métodos: Foi realizado um estudo quantitativo, de abordagem estatística, descritiva e transversal. Resultados: A amostra constituiu-se por 48 docentes. Os resultados mostraram que $75 \%$ dos professores são graduados em medicina. Quanto ao módulo em que atuam, predominaram o "Internato" (41,7\%) e as "Habilidades Clínicas" (29,2\%). Quanto à titulação acadêmica, 35\% apresentavam pós-graduação Stricto Sensu, 29\% mestrado e apenas 6\% doutores, o restante detinha alguma especialização. Com referência ao vínculo empregatício, apenas $44 \%$ dos pesquisados relataram ter vínculo efetivo com a instituição e destes, somente $8 \%$ encontram-se em dedicação exclusiva. Os tutores, em sua maioria (74,1\%) apresentaram pelo menos 2 vínculos empregatícios além do vinculo com a universidade, com variação em até 4 vínculos diferentes. Contatou-se ainda que apenas 29\% dos docentes cursaram alguma pós-graduação voltada para educação e que 60\% não tiveram nenhum treinamento para atuar em metodologias ativas de aprendizagem. Conclusão: Diante dos resultados, sugere-se que se realizem mudanças nas condições de trabalho dos professores, criando-se incentivos, em termos de progressão na carreira, recompensas profissionais, projetos de aprimoramento em educação médica, especializações e bolsas de mestrado e doutorado vinculadas à instituição de ensino, para que a carreira acadêmica torne-se mais atraente e almejada aos olhos desses profissionais.

PALAVRAS-Chave: Educação médica. Aprendizagem baseada em problemas. Capacitação. Condições de trabalho.

\section{ABSTRACT}

The teaching staff is the fundamental basis about which should be instituted necessary changes to the formation of health area. Several studies have noted the absence of pedagogical preparation of the teacher of medicine with regard, in special, to the active learning methodology. Objective: This article traced the professional profile of the medical school professors from Pará State University (UEPA) in Santarém, and know their level of training about medical teaching methodologies, with an emphasis on Problem-Based Learning method (PBL). Methods: This is This is a quantitative study, with descriptive approach, descriptive and transversal. Results: The sample was made up of 48 teachers. The results showed that $75 \%$ of teachers are graduated in medicine. Regarding the module in which they work, they predominated the "Internship" (41.7\%) and "Clinical Skills" (29.2\%). As for the academic degree, 35\% had postgraduate stricto sensu, $29 \%$ master's and only $6 \%$ doctors, the rest detained some specialization. With reference to employment relationship, only $44 \%$ of surveyed reported having effective link with the institution and of these, only $8 \%$ are in exclusive dedication. The tutors mostly $(74.1 \%)$ have at least two employments beyond with the university, ranging by up to 4 different links. It was further observed that only $29 \%$ of teachers studied some postgraduate focused on education and $60 \%$ had no training to work in active learning methodologies. Conclusion: Face of the results, it is 
suggested that there will be changes in the working conditions of teachers, creating incentives, in terms of career development, professional rewards, improvement projects in medical education, specialization and master's and doctorate scholarships linked the educational institution, for the academic career to become more attractive and desired in the eyes of these professionals.

KEYWORDS: Education medical. Problem-based learning. Training. Working conditions.

\section{Introdução}

O corpo docente é a base fundamental sobre a qual devem ser instituídas as mudanças necessárias para a formação da área da saúde, e a prática pedagógica tem se constituído em um tema relevante de investigação na área médica. Nesse contexto, é ainda recente a concepção de que, além das competências específicas para exercer a profissão médica, existem as competências relacionadas especificamente à docência universitária, como o domínio pedagógico para a boa prática docente ${ }^{1}$.

Em estudo recente, foi possível perceber a falta de preparo da maioria dos docentes das escolas médicas ${ }^{1}$. O corpo docente é um dos principais pilares para reformulações necessárias à educação médica, como a implementação do Problem-Based Learning (PBL), que se tornou mais difundido no Brasil com o advento das Diretrizes Curriculares Nacionais (DCN) para o curso de medicina em 2001. Estas diretrizes determinam que os cursos de graduação em medicina devam ter um projeto pedagógico centrado no aluno como sujeito de aprendizagem e apoiado no professor como facilitador e mediador do processo de ensino-aprendizagem, atendendo aos preceitos de aprendizagem ativa ${ }^{1,2}$.

Diante desse contexto, há falta de profissionalização docente, ou seja, domínio de conhecimentos, comportamentos e habilidades para o ensino, e frequentemente se desconhece a estrutura curricular e a relação entre as disciplinas ${ }^{3}$. A instituição de um modelo de aprendizado diferenciado, que rompe com as estruturas curriculares tradicionais do ensino médico, a Aprendizagem Baseada em Problemas (ABP), os desafios pedagógicos para capacitação dos professores são ainda maiores, haja vista o pouco conhecimento prévio que os profissionais possuem sobre a metodologia (a grande maioria não foi sequer formada nela, já que a metodologia foi introduzida recentemente no projeto político pedagógico de algumas escolas médicas brasileiras) ${ }^{4}$.

Como em algumas outras áreas, os docentes de cursos de graduação da área da saúde, especificamente de medicina, veem a docência apenas como uma atividade complementar e secundária. Este fator faz com que os próprios docentes (e até mesmo as instituições) não reconheçam no comprometimento com a universidade, um fator essencial para a prática da docência. Combinado à falta de capacitação pedagógica e uma metodologia de ensino-aprendizagem inovadora, cria-se um cenário peculiar onde o docente pouco reconhece seu papel e não possui preparo didático adequado ${ }^{1,2,4}$.

Portanto, este artigo avaliou o grau de capacitação pedagógica, o nível de comprometimento universitário, bem como o conhecimento teórico acerca da ABP no curso de medicina da Universidade do Estado do Pará, campus Santarém, que emprega, há mais de oito anos, as metodologia ativas como base de seu projeto político pedagógico.

\section{Métodos}

Trata-se de estudo quantitativo, de abordagem estatística descritiva, sendo ainda transversal. Antecipadamente realizou-se uma análise estatística através do programa StatDisc 5.0 para realização do cálculo amostral. Desse modo, foi observado que no universo de 75 professores (concursados e contratados) do curso de medicina da Universidade do Estado do Pará - campus Santarém - o espaço amostral ideal para uma pesquisa significativa, seria com 48 docentes, sendo estes, selecionados 
aleatoriamente. Posteriormente, para análise gráfica foram utilizados os softwares Bioestat $^{\circledR}$ e Excell 2010.

Os critérios de inclusão para a pesquisa foram: a) Docentes do curso de Medicina da Universidade do Estado do Pará em Santarém, sem a discriminação de tipo de atuação docente, vínculo ou formação profissional que aceitassem participar da pesquisa por meio da assinatura do Termo de Consentimento Livre e Esclarecido (TCLE); b) Docentes que não estivessem de licença ou cedidos para outros cursos dentro da Universidade.

A coleta de dados ocorreu seguindo os seguintes passos: a) De comum acordo firmado entre o(s) pesquisadores e o(s) professor (es), através de TCLE, para o preenchimento de questionário; b) fornecimento de questionário, dividido em duas temáticas: (1) 14 questões relacionadas ao seu perfil e formação pedagógica e profissional; (2) 8 questões referentes à percepção de seu conhecimento sobre metodologias ativas de aprendizagem e conhecimentos fundamentais sobre o tema. O questionário contém questões de três vertentes: (1) Perfil acadêmico e profissional do pesquisado: sobre idade, instituição de graduação, vínculo institucional com a universidade, formação acadêmica, titulação, área de atuação médica, experiências profissionais em docência etc. (2) Grau de capacitação pedagógica para a docência: se cursou disciplina de prática pedagógica ou afins, se teve capacitação para docência em ABP entre outros. (3) Conhecimento dos docentes sobre a base filosófica e pedagógica da ABP, baseado na literatura atual, e no Projeto Político-Pedagógico do curso de medicina da UEPA.

O projeto de pesquisa foi submetido à avaliação do Comitê de Ética em Pesquisa com Seres Humanos (CEP) da Universidade do Estado do Pará, Campus XII. Foram obedecidas às normas éticas definidas pela Declaração de Helsinque, Código de Nurenberg e Resolução 466/2012 CONEP. A execução da pesquisa só ocorreu após a devida aprovação pelo CEP sob o parecer de número 647.106 (registro no Sisnep de $\mathrm{n}^{\mathrm{o}}$ 30306314.2.0000.5168).

\section{Resultados}

Participaram da pesquisa 48 indivíduos, do total de 75 docentes, com idade média de 42,5 anos (variando de 29 a 63) e mediana de 46 anos. Destes, 36 (75\%) eram médicos e 12 (25\%) não médicos. A maioria dos profissionais foi graduada na Universidade Federal do Pará (20), na Universidade do Estado do Pará (6) e oriundos de outras instituições somaram 22.

Participaram da pesquisa professores de todos os períodos do curso, sendo que a maioria afirmou fazer parte do quadro de docentes do eixo "Internato". Contudo, 08 docentes consideraram sua atuação em mais de um módulo do curso. Isso ocorre graças à sua inserção concomitante em eixos do ciclo "básico" e do "internato" durante suas atividades, devido à indissociável prática-teoria, que é imprescindível em metodologias ativas de aprendizagem (Gráfico 1).

Quanto à formação acadêmica, apenas 35\% dos participantes declararam ter título de mestrado ou doutorado (Tabela 1). Já a grande maioria dos docentes (65\%), apresentava somente alguma especialização.

Em relação aos vínculos profissionais e empregatícios dos docentes, representado no Gráfico 2, apenas 44\% (21) têm vínculo efetivo com a instituição, o que traz a tona a instável realidade dos docentes do curso de Medicina da Universidade do Estado do Pará em Santarém, por falta de maiores vínculos, incentivos e compromissos institucionais com o profissional.

Com relação ao número de empregos além da carreira docente, somente $8 \%(n=4)$ declararam estar em regime de dedicação exclusiva; os outros quase $92 \%$ relataram necessitar de mais empregos para melhor estabilidade financeira. 


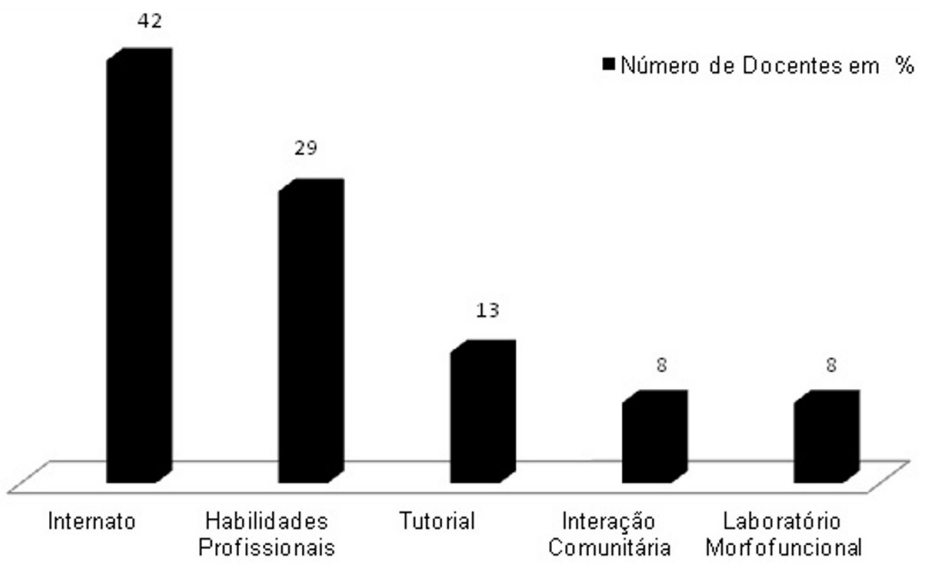

Gráfico 1. Módulo de atuação dos docentes do Curso de Medicina da Universidade do Estado do Pará.

- Tipo de vínculo com a Instituição \%

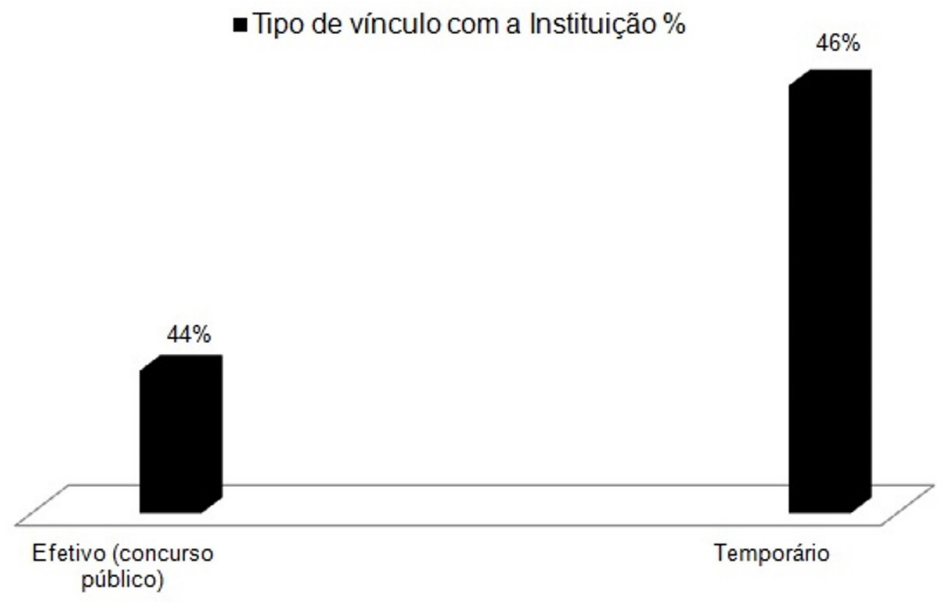

Gráfico 2. Tipo de vínculo empregatíco entre o docente e a UEPA em porcentagem.

Tabela 1. Titulação acadêmica dos docentes da Universidade do Estado do Pará-UEPA.

\begin{tabular}{ccc} 
& \multicolumn{3}{c}{ Titulação Acadêmica } \\
\cline { 2 - 3 } & $N^{\circ}$ & $\%$ \\
\hline Especialização & 31 & 65 \\
\hline Mestrado & 14 & 29 \\
\hline Doutorado & 3 & 6 \\
\hline Total & 48 & 100 \\
\hline
\end{tabular}

Na Tabela 2, evidencia-se que $29 \%$ dos docentes cursaram alguma pós-graduação voltada para educação, contudo, essa porcentagem ainda é pequena, posto que 14 professores não tiveram qualquer treinamento voltado para sua função dentro da instituição. Outro dado preocupante, é que a maioria dos professores do curso de medicina não teve capacitação para atuar em metodologias ativas de aprendizagem e nem vivenciaram como discente essa metodologia, representando, respectivamente, $60 \%$ e $62,5 \%$ dos docentes da UEPA.

Em relação ao conhecimento dos docentes sobre diversas variáveis que englobam o ensino médico (Tabela 3), como as DCN, o plano político pedagógico do curso de medicina (PPP), ementa do módulo de sua atuação e o conhecimento sobre a metodologia - ABP, a maioria dos docentes afirmou conhecer ou conhecer parcialmente. Contudo, vale ressaltar, que um expressivo número de professores 
Tabela 2. Número e porcentagem de docentes que cursaram pós-graduação em educação e "vivência" em metodologias ativas de aprendizagem.

\begin{tabular}{|c|c|c|c|c|c|c|}
\hline \multirow[t]{2}{*}{ Categoria } & \multicolumn{2}{|c|}{$\begin{array}{c}\text { Cursou pós-graduação voltada } \\
\text { à educação }\end{array}$} & \multicolumn{2}{|c|}{$\begin{array}{c}\text { Recebeu capacitação para atuar } \\
\text { em metodologias ativas de } \\
\text { aprendizagem }\end{array}$} & \multicolumn{2}{|c|}{$\begin{array}{c}\text { Já foi discente em metodologias } \\
\text { ativas de aprendizagem }\end{array}$} \\
\hline & $N^{o}$ & $\%$ & $N^{o}$ & $\%$ & $N^{o}$ & $\%$ \\
\hline Sim & 14 & 29 & 19 & 39,5 & 18 & 37,5 \\
\hline Não & 34 & 71 & 29 & 60,5 & 30 & 62,5 \\
\hline Total & 48 & 100 & 48 & 100 & 48 & 100 \\
\hline
\end{tabular}

Tabela 3. Porcentagem do grau de conhecimento dos docentes sobre variáveis do ensino.

\begin{tabular}{cccc} 
Categorias & Sim $\%$ & Parcialmente $\%$ & Não \% \\
\hline Conhece as DCN de Medicina & 21 & 66,5 & 12,5 \\
\hline Conhece o PPP & 46 & 39,5 & 14,5 \\
\hline Conhece a ementa do seu módulo & 51,5 & 40 & 8,5 \\
\hline Conhece bem a ABP & 25 & 44 & 31 \\
\hline
\end{tabular}

(31\%), desconhecem a metodologia - Aprendizagem Baseada em Problemas (ABP) que fundamenta o curso de medicina na Universidade do Estado do Pará (campus Santarém).

\section{Discussão}

Neste estudo, os professores foram questionados a respeito de suas habilidades em diversos aspectos da docência, não apenas quanto a capacidade individual de lecionar, mais além: quanto ao poder de se adequar as perspectivas de um método ainda novo na realidade da Universidade do Estado do Pará, um método que se sustenta na busca ativa por conhecimento, mas que tem como um de seus pilares a figura do tutor, aquele que fomenta a busca pelo conhecimento, orienta como fazê-lo e também avalia².

Dentre os aspectos pesquisados, encontra-se a universidade na qual o docente realizou sua graduação e o contato prévio com metodologias em outras universidades, tanto na qualidade de discente como de docente. Os resultados evidenciam que a maioria dos mentores teve sua iniciação e seu primeiro contato com a ciência médica por meio do método tradicional, bem como suas primeiras experiências como professores nesse mesmo método e, ao longo de vários anos, lecionaram tendo essa metodologia como base.

Esse fato, revela o distanciamento dos professores de medicina em relação as novas abordagens do ensino médico, sendo esse, recorrentemente, o principal obstáculo à atualização pedagógica no curso. Feuerwerker em sua obra intitulada "Além do discurso de mudança na educação médica: processos e resultados": relata que a prática docente em medicina tem se mostrado resistente a modificações, visto que os professores continuam a ensinar como sabem - saber este, muitas vezes provindo da imitação prática dos mais experientes - e resistem a novas metodologias de ensino-aprendizagem ${ }^{5}$.

As escolas médicas apresentam, em geral, organizações tradicionais, embasadas em grupos de poder hierarquizados, departamentos desarticulados, os quais terminam por promover um aprendizado em que mestres (sabedores) orientam 
discípulos (receptores de informação) em rituais de poder e hierarquia, relações que se reproduzem e se perpetuam na atenção à saúde ${ }^{6,7}$.

Diante dessa perspectiva, as iniciativas sugeridas pelas DCN e Promed acabam - de certa maneira - "confrontando" essas práticas, na medida em que incentivam as atividades de ensino que capacitem os novos médicos, desde os períodos mais tenros, a exercerem a medicina de modo autonômico ao longo da vida. Discordando assim, da tradicional e estabelecida ideia, de que e papel exclusivo do professor fundamenta-se em esclarecer sobre as funções profissionais e o modo como essas devem ser desempenhadas ${ }^{2}$.

A mudança de enfoque, do ensino para a instrução de aprendizagem, exige do mentor todo um novo conjunto de competências de natureza pedagógica ${ }^{8}$. Ocorre uma transposição didática inicial: "dos saberes de referência para objetos de ensino" e posterior: "dos objetos de ensino para os objetos de aprendizagem". Há também uma atenção para este ensino superior "novo", onde se vive uma mudança de paradigma e em que o objetivo da universidade passou a ser mais o de produzir aprendizagem, deixando em segundo plano o "dar" instruções ${ }^{9,10}$.

Identificou-se também, o predomínio de mentores dedicando-se a apenas um módulo, sendo esse ou "habilidades clínicas" ou "internato". Este resultado revela de modo prático, a tendência do ensino médico brasileiro em criar profissionais que atuem em apenas um departamento de modo isolado o qual, na maioria das vezes, torna-se hipervalorizado pelo profissional em detrimento das outras áreas de atuação. Margeando assim, a proposta global de ensino proposta nas metodologias ativas de aprendizagem, a qual propõe o direcionamento a uma prática médica completa e antes dessa: um saber pedagógico completo ${ }^{1,9}$.

O professor necessita, portanto, do contato mais hibridizante com a academia, para que não perca a noção da universalidade do estudo além da sua área de atuação. $\mathrm{O}$ motivo desta discordância provém do modelo acadêmico admitido tradicionalmente na maioria das universidades brasileiras, a qual tende a valorizar em demasia, a produção acadêmica e formação científica do profissional, a despeito de sua destreza pedagógica, ignorando assim um dos pilares da universidade, o ensino.

A atribuição de pesos diferentes às duas principais funções do professor de ensino superior - investigar e ensinar - implica que o prestígio profissional dos docentes decorra, quase exclusivamente, da atividade de investigação e de produção científica e que a dedicação à investigação determine o acesso à estabilidade profissional, muitas vezes em detrimento da atividade docente ${ }^{10}$.

Quanto às titulações, a maioria dos professores (65\%) revelaram ter apenas especialização em sua área de atuação médica, sem nunca ter tido contato continuado com disciplinas referentes a pedagogia universitária. Sendo o conhecimento dessa disciplina, adquirido ou em forma de cursos de pequena duração, ou sem mesmo nunca ter estudado formalmente nada referente a esse assunto.

Em estudo realizado na Universidade Federal de Goiás, sobre a qualificação em docência (ou falta dela) dos professores de medicina, foi observado os seguintes relatos: "Foi uma aquisição pela experiência, um autodidatismo"; "muita improvisação, nenhuma base teórica"; "fazia as coisas mais intuitivamente"; "nós não temos formação em docência, nossa formação é medica"; " Tinha a preparação médica, no entanto, não tinha nem uma preparação em docência"; " Fui equivocando-me e acertando"; " Gosto muito de ensinar, então penso que é por afinidade e experiência". Esses professores, de modo geral, são considerados bons profissionais em sua área especifica de atuação e o critério de contratação baseia-se exatamente nesta prerrogativa, isto é, na qualidade de desempenho na área técnica de atuação ${ }^{11}$.

Este estudo demonstrou ainda que menos da metade tem vínculo efetivo com a instituição e apenas $8 \%$ trabalham em regime de dedicação exclusiva, além de em sua maioria terem 2 ou 3 vínculos empregatícios além da atividade exercida na 
universidade. Sendo a deficiência nos vínculos trabalhistas efetivos e a diversidade de modalidades de vínculos empregatícios - "horistas", "substitutos", a principal razão pelos quais à docência é, geralmente, considerada uma atividade secundária à profissão médica, e a carreira de docência não é vista como uma única profissão ${ }^{12,13}$.

Outra dificuldade que merece destaque, são as variadas tarefas que o tutor desempenha no seu dia dia, por estabelecer diversos vínculos empregatícios e ter que cumprir todas as atividades de uma agenda repleta de compromissos fora da academia ${ }^{14}$.

Nesta análise, vale notar ainda, que os poucos professores que chegaram a obter preparo pedagógico (menos de $40 \%$ dos pesquisados), em sua maioria obtiveram instruções insuficientes e de caráter instrumental e genérico, por vezes limitados à experiência e à intuição, calcado nos modelos vivenciados por eles na qualidade de aluno, em sua maioria nos modelos tradicionais ${ }^{15-17}$.

As barreiras mais frequentes na implantação de mudanças curriculares se referem à "falta de professores com a devida habilitação pedagógica, estruturas curriculares tradicionais e a ausência de espaços específicos para esse ensino" ${ }^{\prime 1}$.

Em relação aos perfis de outras escolas, temos: um menor percentual de titulados mestres e doutores (35\% para 47,9\% na Universidade Estadual de Maringá-UEM e 25\% na Universidade Federal de Santa Maria-UFSM. Na UEM, por exemplo, 52,2\% dos professores não apresentam pós-graduação stricto sensu, enquanto na universidade pesquisada (UEPA) este percentual representa $65 \%$ do total de participantes; na UEM $31,9 \%$ finalizaram o mestrado e $15,9 \%$ o doutorado e $16,8 \%$ referiram cursos isolados de capacitação pedagógica, estes números na Universidade do Estado do Pará representam, respectivamente $29 \%, 6 \%$ e $39 \% 18,19$.

Os números inferiores encontrados neste estudo em relação a outras universidades, devem-se provavelmente a ainda recente implantação do curso no campus do interior e aos ainda incipientes projetos de pós-graduação ofertados ao mesmo. Portanto, a tendência atual é que o número de pós-graduados e o nível de complexidade de suas respectivas pós-graduações sejam, gradativamente, elevados, à medida em que aumentem as vagas ofertadas ao interior ${ }^{18,19}$.

Quanto ao conhecimento sobre as diretrizes curriculares nacionais em educação médica, o Projeto Político pedagógico do curso e a ementa do curso em que atuam, os resultados apresentaram que menos de $10 \%$ dos professores consultados tinham conhecimento da completude desses documentos e que menos da metade os conheciam parcialmente. Essa constatação se justifica por meio dos resultados anteriores, os quais registram diversos fatores complicadores, como a falta de compromisso de parte dos docentes, as dificuldades destes em se adequarem aos novos moldes pedagógicos, à dedicação parcial à escola, à adoção de uma postura de indiferença ${ }^{20}$.

Quanto ao PPP, destacando a sua função integradora, no sentido de que aproxima fundamentos práticos; atualizadora, no sentido de que constitui objeto de reavaliações desses fundamentos e práticas; e estruturante, no sentido de que articula e consolida a constituição e perspectivas acadêmicas dos cursos. Partindo da premissa do autor, portanto, a falta do conhecimento do PPP desfavorece a consciência do sentido do curso, fadando suas propostas, objetivos e conceitos fundamentais ao esquecimento ${ }^{21}$.

\section{Conclusão}

As constatações desse estudo demonstram e reafirmam os passos lentos por meio dos quais o saber pedagógico universitário e as metodologias de ensino médico, destacadamente as metodologias ativas, caminham rumo as atualizações e mudanças efetivas que permitam a adequação às DCN. 
Espera-se que as necessidades constatadas sirvam de base para elaboração de ferramentas que visem incrementar e modificar a realidade educacional vigente nos cursos de medicina em geral. Sugere-se assim, que a academia desenvolva novas abordagens para instigar nos professores a aquisição de novas competências e de novos conhecimentos e que, estudos como este, sirvam de oportunidades para eles refletirem criticamente sobre as suas práticas e para adaptarem, aos seus contextos, novos conhecimentos e novas formas de trabalho. Pois é evidente que a formação médica é um processo de apropriação individual que se faz numa permanente interação, e o tutor é parte indissociável desse processo.

Ademais, é valido propor que seja despendido maior tempo e atenção aos conteúdos essenciais do curso de Medicina, que estes sejam instituídos a partir da análise do processo saúde-doença individual, porém sem esquecer a realidade epidemiológica do país, e que estas modificações impliquem na integração docente-assistencial. Reforçando deste modo, o compromisso da universidade com a efetivação do Sistema Único de Saúde (SUS), ao formar novos médicos atuantes em todos os níveis do sistema.

Por fim, destaca-se a necessidade de uma maior atenção as mudanças relacionadas as condições de trabalho do professor, para que esta carreira torne-se mais atraente. Devem-se criar incentivos, em termos de progressão na carreira, recompensas profissionais, projetos de aprimoramento em educação médica, especializações e bolsas de mestrado e doutorado vinculadas à instituição de ensino. Pois torna-se óbvio, que somente com a valorização do professor, o sistema poderá vislumbrar sair do contexto atual de crise e insatisfação generalizada tanto com a academia quanto com a prática médica em geral.

\section{Referências}

1. Costa NMSC. Docência no ensino médico: por que é tão difícil mudar? Rev Bras Educ Med. 2007;31(1):21-30. http://dx.doi. org/10.1590/S0100-55022007000100004.

2. Brasil. Ministério da Educação. Conselho Nacional de Educação. Câmara de Educação Superior. Resolução $\mathrm{CNE} / \mathrm{CES} \mathrm{n}^{\circ} 4$ de 7 de novembro de 2001. Institui diretrizes curriculares nacionais do curso de graduação em Medicina. Diário Oficial da União. Brasília, 9 nov. 2001; Seção 1, p. 38.

3. Garcia MAA, Silva ALB. Um perfil do docente de medicina e sua participação na reestruturação curricular. Rev Bras Educ Med. 2011;1(35):58-68. http://dx.doi.org/10.1590/S0100-55022011000100009.

4. Allen DE, Donham RS, Bernhardt SA. Problem-based Learning. New Directions for Teaching and Learning [Internet]. 2011 [citado em 2014 jun. 5]. no. 128. Disponível em: http://wileyonlinelibrary.com

5. Feuerwerker L. Além do discurso de mudança na educação médica: processos e resultados. Rio de Janeiro: ABEM; 2002.

6. Batista NA. A formação do professor de medicina: desafios e perspectivas. São Paulo: Sarvier; 1998.

7. Rodríguez CA, Poli Neto P, Behrens MA. Paradigmas educacionais e a formação médica. Rev Bras Educ Med. 2004;28(3):234-41.

8. Cachapuz A, Praia J, Jorge M. O caso do docente médico. Porto: Ciência \& Educação; 2004.

9. Cross KP. Leading-edge efforts to improve teaching and learning. The Magazine of Higher Learning. 2001;33(4):30-7.

10. Dill DD. (2003). An institutional perspective on higher education policy: the case of academic quality assurance. In: Smart JC, editor. Higher education: handbook of theory and research (pp. 669-700). Dordrecht: Kluwer Academic Publishers. http://dx.doi.org/10.1007/978-94-010-0137-3_12.

11. Costa NMSC. La formación pedagógica de profesores de medicina. Rev Lat Am Enfermagem. 2010;18(1):1-7.

12. Bireau A. Os métodos pedagógicos no ensino superior. Porto: Porto Editora; 1995.

13. Mclean M. Reawarding teaching excellence. Can we measure teaching "excellence"? Who should be the judge? Med Teach. 2001;23(1):6-11. http://dx.doi.org/10.1080/01421590123039.

14. Bellodi PL, Cardillo GZ. O aluno da FMUSP e seus colegas [Internet]. 2015 [citado em 2015 Jun 9]. Disponível em: http:// www.fm.usp.br/cedem/peqedu/perfil20005.php

15. Lampert JB. Avaliação do processo de mudança na educação médica. São Paulo: Hucitec; 2004.

16. Batista NA, Silva SHS. O professor de medicina: conhecimento, experiência e formação. São Paulo: Loyola; 1998.

17. Abreu IP No, Lima OS Fo, Silva LEC, Costa NMSC. Percepção dos professores sobre o Novo Currículo de Graduação da Faculdade de Medicina da UFG, implantado em 2003. Rev Bras Educ Med. 2006;30(3):154-60.

18. Moré NC, Gordan PA. A percepção dos professores do Departamento de Medicina da Universidade Estadual de Maringá sobre suas dificuldades e necessidades educacionais para o desenvolvimento do ensino médico. Rev Bras Educ Med. 2004;28(3):215-22. 
19. Souza OC Fo, Naujorks MI. O professor de Medicina da UFSM no contexto do mal-estar docente. Rev Bras Educ Med. 2005;29(1):34-41.

20. Marcondes E, Gonçalves EL. Educação médica. São Paulo: Sarvier; 1998.

21. Pinto LAM, Rangel M. Projeto político-pedagógico da escola médica. Rev Bras Educ Med. 2004;28(3):251-58.

\section{Contribuição dos autores}

Todos os autores participaram do planejamento e execução do trabalho. 